\title{
A Web-Based Skin Disease Diagnosis Using Convolutional Neural Networks
}

\author{
Samuel Akyeramfo-Sam \\ Department of Computer Science, Sunyani Technical University, Sunyani Ghana \\ E-mail: samatosam519@gmail.com \\ Acheampong Addo Philip \\ Department of Computer Science, Sunyani Technical University, Sunyani Ghana \\ E-mail: philinzag.edu@gmail.com \\ Derrick Yeboah \\ Department of Computer Science, Sunyani Technical University, Sunyani Ghana \\ E-mail: drrickyeboah999@hotmail.com \\ Nancy Candylove Nartey \\ Department of Computer Science, Sunyani Technical University, Sunyani Ghana \\ E-mail: nahcey88@gmail.com \\ Isaac Kofi Nti \\ Department of Computer Science, Sunyani Technical University, Sunyani Ghana \\ E-mail:ntious1@gmail.com
}

Received: 07 June 2019; Accepted: 28 September 2019; Published: 08 November 2019

\begin{abstract}
Skin diseases are reported to be the most common disease in humans among all age groups and a significant root of infection in sub-Saharan Africa. The diagnosis of skin diseases using conventional approaches involves several tests. Due to this, the diagnosis process is seen to be intensely laborious, time-consuming and requires an extensive understanding of the domain. The enhancement of computer vision through artificial intelligence has led to a more straightforward and quicker way of detecting patterns in images, which can be harnessed to equip diagnosis process. Despite the breakthrough in technology, the dermatological process in Ghana is yet to be automated, making the diagnosis process complicated and time-consuming. Hence, this study sought to propose a web-based skin disease detection system named medilab-plus using a convolutional neural network classifier built upon the Tensorflow framework for detecting (atopic dermatitis, acne vulgaris, and scabies) skin diseases. Experimental results of the proposed system exhibited classification accuracy of $88 \%$ for atopic dermatitis, $85 \%$ for acne vulgaris, and $84.7 \%$ for scabies. Again, the computational time (0.0001 seconds) of the proposed system implies that any dermatologist, who decides to implement this study, can attend to not less than 1,440 patients a day compared to the manual diagnosis process. It is estimated that the proposed system will enhance accuracy and offer fasting diagnosis results than the traditional method, which makes this system a trustworthy and resourceful for dermatological disease detection. Additionally, the
\end{abstract}

system can serve as a realtime learning platform for students studying dermatology in medical schools in Ghana.

Index Terms-Skin disease detection, Expert-system, Convolutional neural network, Tensorflow, Atopic dermatitis, Acne vulgaris, Scabies.

\section{INTRODUCTION}

The human skin plays a huge part in a person's physical appearance, and it is the biggest organ of the human body. The human-skin offers protection against fungal infection, bacteria, allergy, viruses and controls the temperature of the body situations that change the texture of the skin or damage the skin can produce symptoms like swelling, burning, redness, and itching [1]. Antipathies, genetic structure, irritants, and particular diseases and immune system associated complications can produce hives, dermatitis, and other skin problems. Many of the skin diseases, such as alopecia, eczema, acne, ringworm, also affect a person's look. Skin diseases are widespread these days; some of them are simple and easy to recover from; others are very harmful and might be incurable, and many of these diseases are very dangerous, mainly if not treated in the early stages.

In literature, dermatological diseases are reported to be the most widely spread disease [2]. A survey by Hogewoning et al. [3] revealed that the total frequency of 
pupils with some skin disease was $34.6 \%$ and $42.0 \%$ in two (2) Ghanaian studies, out of 4,839 pupils surveyed. Again, in [4] reports, out of 529 participants surveyed, 700 discrete skin diagnoses were made [4]. Therefore, detection of skin disease at its early stages is paramount to its spreading.

On the other hand, skin disease diagnosis is seen to be complicated, mainly when two or more diseases portray same or similar symptoms, hence requires a dermatologist with vast experience of skin diseases $[2,4]$. Nevertheless, the development in technology and machine learning have changed all aspects of one's dayto-day life, including the medical field [5,6]. Many therapeutic systems have been developed with the help of artificial intelligence (AI) and technological advancement to help both doctors and patients in diverse ways, starting from Out Patient Department (OPD), consultation to the operating theatre or operating room (OR). Thus, the introduction of artificial intelligence into the health industries has brought tremendous improvement in the diagnoses of skin disease and other illness [7].

However, in Ghana, most dermatologists still use a variety of manual visual clues such as colour, scaling, and arrangement of the lesions, the body site distribution, among others. Nonetheless, when these individual components are analysed separately, the recognition of the disease can be quite complex, thus requiring a high level of experience. Human diagnosis is based on a subjective judgment of the dermatologist, so it is hardly reproducible, unlike computer-aided diagnostic systems, which are more realistic and reliable.

To reduce diagnosis time and provide quick health service, some researchers in recent years proposed skin disease detection system with the ability to detect skin disease like impetigo, eczema, melanoma and acne using machine learning [8-10]. On the other hand, these skin diseases are not prevalent in Ghana, as indicated in [4].

Furthermore, Ghana currently has only one dermatology-training centre at the Korle $\mathrm{Bu}$ Teaching Hospital (KBTH), with only four (4) dermatologists three
(3) on full-time and one (1) part-time, and three (3) trainees. In reality, the total number of dermatologists serving the whole people of Ghana is lesser than 25 [4]. On average, a patient with skin disease spent not less than two hours in a medical centre.

Finally, with a population 30,030,189 as of May 2019 [11], it implies that every dermatologist in Ghana is to $1,201,207.56$ patients.

In an attempt to reduce issues mentioned above, the current study seeks to develop a smart web-based skindisease detection system (medilab-plus) for faster and reliable early detection of atopic dermatitis, acne vulgaris, and scabies, using the convolutional neural network (CNN).

The development of the proposed system will offer foreknowledge, quick and faster diagnosis system to users through the internet. Again, serve as the first skin diseases system built and tested with sample data from Ghana.

The remaining section of this study is categorised as follows: Section 2 present review of common skin diseases in Ghana, the application of machine learning in disease diagnosis system, and related studies. Section 3 covers the methods, tools and evaluation metrics adopted for the current study. Section 4 presents the outcome and discussion of the study. Finally, Section 5 concludes the study and the direction for future studies.

\section{LITERATURE REVIEW}

This section gives a brief discusses of common skindisease in Ghana, the application of machine learning in disease diagnosis system, and related studies.

\section{A. Skin diseases in Ghana}

There are numerous types of skin diseases identified in Ghana. However, table 1 shows the common skin diseases in Ghana and their prevalence among males and females, as presented in [4].

Table 1. Common skin diseases in Ghana

\begin{tabular}{|c|c|c|c|c|c|}
\hline Overall $(N=700)$ & $\mathbf{N}(\%)$ & Males $(N=302)$ & $\mathbf{N}(\%)$ & Females $(N=396)$ & $\mathbf{N}(\%)$ \\
\hline Atopic dermatitis & $59(8.4)$ & Atopic dermatitis & $24(7.9)$ & Atopic dermatitis & $35(8.8)$ \\
\hline Acne vulgaris & $37(5.3)$ & Scabies & $17(5.6)$ & Pityriasis rosea & $21(5.3)$ \\
\hline Scabies & $36(5.1)$ & Warts & $17(5.6)$ & Lichen planus & $21(5.3)$ \\
\hline Irritant contact dermatitis & $33(4.7)$ & Acne vulgaris & $16(5.3)$ & Acne vulgaris & $20(5.1)$ \\
\hline Lichen planus & $26(3.7)$ & Irritant contact dermatitis & $14(4.6)$ & Scabies & $19(4.8)$ \\
\hline Seborrhoeic dermatitis & $25(3.6)$ & Seborrhoeic dermatitis & $11(3.6)$ & Irritant contact dermatitis & $19(4.8)$ \\
\hline Warts & $23(3.3)$ & Tinea pedis & $10(3.3)$ & Vitiligo & $14(3.5)$ \\
\hline Vitiligo & $22(3.1)$ & Pityriasis versicolor & $9(3.0)$ & Papular urticaria & $13(3.3)$ \\
\hline Pityriasis versicolor & $17(2.4)$ & Chronic urticaria & $9(3.0)$ & Seborrhoeic dermatitis & $13(3.3)$ \\
\hline
\end{tabular}

\section{B. Methods of identifying skin diseases}

Readily visible changes of the skin surface have been recognised since the genesis of history, with some treatable, and some not. In developing countries, overcrowding and poor hygiene are responsible for spreading of skin diseases. One of the known initially sources detailing skin diseases is the Ebers Papyrus, a medical paper from antique Egypt dating to around 1500 BC. It offers descriptions of the various skin diseases, including ulcers, rashes, and tumours, and prescribes surgery and ointments to treat the ailments [12]. There are two ways of detecting or diagnosing skin disease

The first method is the traditional method, also known 
as the conventional method in which skin diseases are detected based on unique colour space. Due to the mixing of chrominance and luminance data, RGB is not the right choice for detection. Although it avoids this problem, its actual detection effect is still unstable and susceptible to some environmental influences $[13,14]$. The specific positioning of the affected area is necessary to detect the type of skin disease.

The second method is the technological method, with the emergence of machine learning, diagnosing of skin disease has become easy for most dermatologists. Computer Vision (CV), Machine-Learning, and Artificial Intelligence are the approach introduce on clinically evaluated histopathological attributes to identify the condition accurately. Firstly, the image is pre-processed, followed by feature extraction. The second stage involves the use of machine-learning algorithms to classify conditions based on the histopathological attributes observed on the analysing of the skin.

A CV is an interdisciplinary field that concerns with how computers can be made to gain a high-level understanding from digital images and videos. From the engineering perspective, it seeks to automate tasks that the human visual system can do. Sub-domains of CV include scene reconstruction, event detection, video tracking, object recognition, object pose estimation, learning, indexing, motion estimation, and image restoration $[1,6]$.

\section{Machine Learning (ML)}

ML is a subcategory of artificial intelligence, which, uses statistical and computational tools to offer humanlike abilities to computers [17,18]. Thus, ML offers automation and enhancement of the learning process of machines based on their experiences without being programmed (no human assistance) [19]. ML Techniques can be grouped into three main categories, namely; supervised, unsupervised, and reinforcement learning [20,21]. ML algorithms such as decision trees (DT), artificial neural network (ANN) support vector machines (SVM), Naïve Bayes and AdaBoost has been applied in the various disease-diagnosis system [1,8,22]. Fig. 1 shows a comparison of accuracy between some machine learning algorithms in disease detection from 2009 to 2015 .

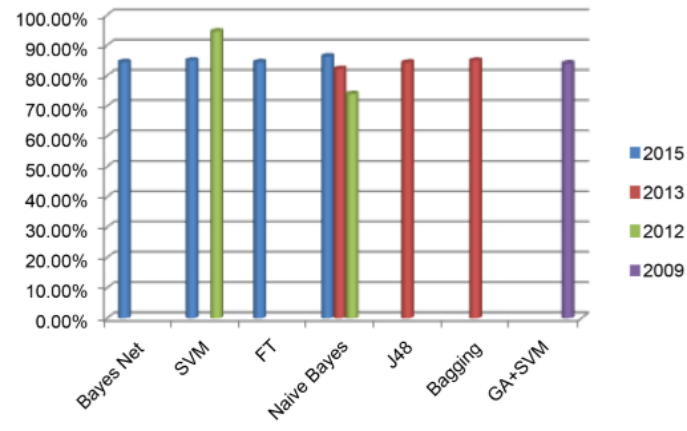

Fig.1. Accuracy of machine learning algorithms in disease detection (source: [22]).

\section{Related works}

A skin disease diagnosis system was proposed in [8], where a user uploads an image of the affected area of the skin into an online system and receive treatment or a piece of advice in a short time. An empirical result of their system offered an accuracy of $95 \%$ for Impetigo, $85 \%$ for Eczema, and $85 \%$ for Melanoma.

An Android app (skinvision) for the diagnosis of melanoma skin disease was proposed in [15]. The accuracy level of the skinvision app was $81 \%$. Users of the app take an image of the disease spot with a phone camera and upload it into the app, and a verdict is given within 30 seconds as low, medium, or high risk. A Melanoma skin cancer detection model was proposed in [9], using a support vector machine.

Similarly, Rathod et al. [2] proposed an application for detecting five (5) different skin disease using convolutional neural networks. The proposed system attained an accuracy of $70 \%$. However, the authors concluded that using a higher dimensional dataset can increase the accuracy above $90 \%$ [2].

Table 2 shows a summary of related studies. Most previous studies were based on the classification of Melanoma and Eczema [8-9] and [16]. Only a few studies [10] were carried out on acne detection. However, these diseases are not prevalent in Ghana as discussed in section 2.1 .

Table 2. Compilation of Related Methods

\begin{tabular}{|c|c|c|c|c|c|}
\hline Reference & Approach & Disease & $\begin{array}{c}\text { Programming } \\
\text { language }\end{array}$ & $\begin{array}{c}\text { Machine language } \\
\text { algorithm }\end{array}$ & Country \\
\hline [8] & Web-based & $\begin{array}{l}\text { Eczema, melanoma, } \\
\text { Impetigo }\end{array}$ & Note stated & Not stated & Not stated \\
\hline [15] & Mobile App (IOS) & Skin cancer & $\begin{array}{c}\text { Python, Java and } \\
\text { swift }\end{array}$ & $\begin{array}{c}\text { k-nearest network } \\
\text { algorithm }\end{array}$ & $\begin{array}{c}\text { United } \\
\text { Kingdom } \\
\end{array}$ \\
\hline [16] & Mobile App & Melanoma & & k-nearest network & Holland \\
\hline [9] & Not stated & Melanoma, Eczema & & Support Vector Machine & India \\
\hline [10] & Android app & $\begin{array}{c}\text { Melanoma, eczema, } \\
\text { acne }\end{array}$ & Matlab, Java & $\begin{array}{c}\text { K-means clustering } \\
\text { algorithm }\end{array}$ & \\
\hline [2] & & Not stated & Python 3.5 & $\begin{array}{c}\text { Convolutional neural } \\
\text { network }\end{array}$ & Not stated \\
\hline Proposed System & Web-based & $\begin{array}{l}\text { Atopic dermatitis Acne } \\
\text { vulgaris and Scabies. }\end{array}$ & Python & $\begin{array}{c}\text { Convolutional neural } \\
\text { network }\end{array}$ & Ghana \\
\hline
\end{tabular}




\section{METHODOLOGY}

This section discussed the methods and materials for the implementation of the proposed skin-disease diagnosis system. Fig. 2 shows the workflow diagram of the proposed classification model. The workflow diagram is divided into three (3); the first discusses the data collection, the second phase deals with the preprocessing of images and learning of the model, and third part involves the classification task and performance measure. The obtained images are passed through image enhancement techniques, and the required features are extracted afterwards. The extracted features were subdivided into three sets, thus train, test, and validation datasets, as shown in Fig. 2. The training dataset was used to trained the $\mathrm{CNN}$ model, and the pattern learned is used for making classification on the test dataset. The accuracy of the model is measured at the evaluation stage, as shown in Fig. 2, to examine the correct and wrong classification rate of the proposed system.

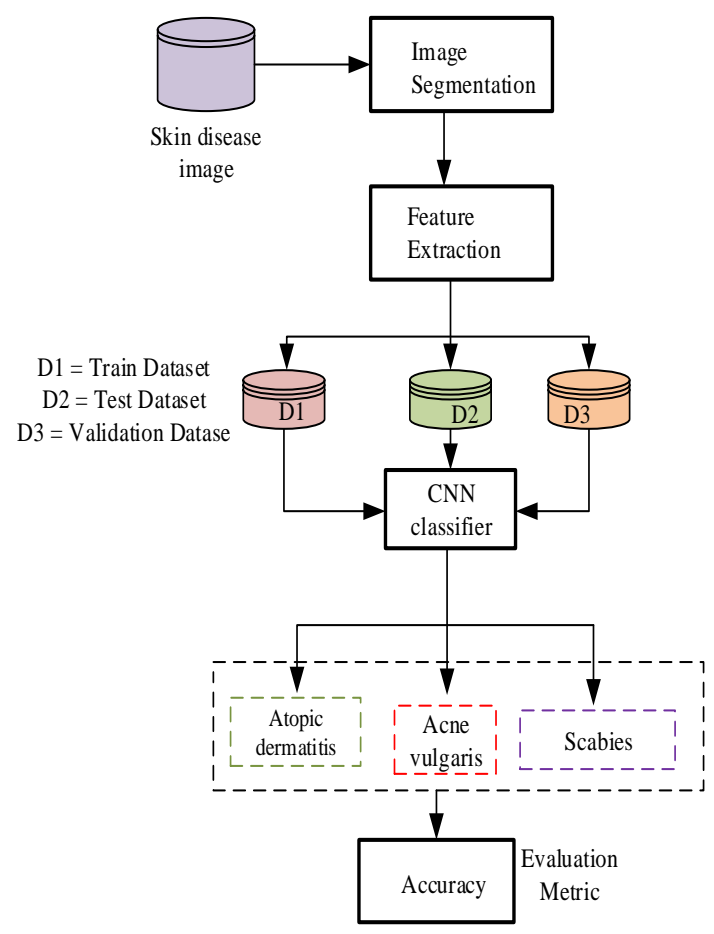

Fig.2. The workflow of the proposed system

\section{A. Dataset and Data preprocessing}

Three diseases, namely: atopic dermatitis, acne vulgaris, and scabies based on their prevalence among the Ghanaian population, as discussed in section 2.1, were selected for this study. The sample data (images) used for this study were collected from four (4) medical centres in the Sunyani Municipality, Ghana, within 30 days. Two hundred and fifty images were used for the current study. Data preprocessing contributes a lot to the accuracy of the machine learning model. The obtained dataset was preprocessed through data cleaning techniques such as smoothing, aggregation normalisation, and attribute construction. Table 3 shows the distribution of the collected dataset for this study.

Table 3. Dataset Size

\begin{tabular}{|c|c|c|}
\hline Skin Diseases & Data sample & Percentage \\
\hline Atopic dermatitis & 102 & $40.16 \%$ \\
\hline Acne vulgaris & 87 & $34.25 \%$ \\
\hline Scabies & 65 & $25.59 \%$ \\
\hline & 254 & $100 \%$ \\
\hline
\end{tabular}

Image Preprocessing: Image processing, as explained in the literature, is the technique of detection and exploring the various images out there and providing the desired output within the type of pictures or different elaborate report [23]. Firstly, the acquired images were preprocessed and converted to a standard size (120X120) to enhance the image quality and to improve the accuracy of the proposed model for better generalisation. Features such as hairs and pigments, which are typically regarded as noisy, were filtered off to facilitate the separation of the lesion area from the surrounding skin.

Image Segmentation: the preprocess images were segmented into disjoint regions that are homogeneous concerning a chosen property such as luminance, colour, and texture. At this stage, the goal was to simplify and change the representation of an image into something more meaningful and more comfortable to analyse.

Feature Extraction: feature selection is an essential part of machine learning since the performance of a machine learning model is much dependent on this phase [24]. The dataset was finally passed through this phase to select the features for the predictive model. For clinical purposes, it is arguable that parsimony is a desirable feature of a good predictive model.

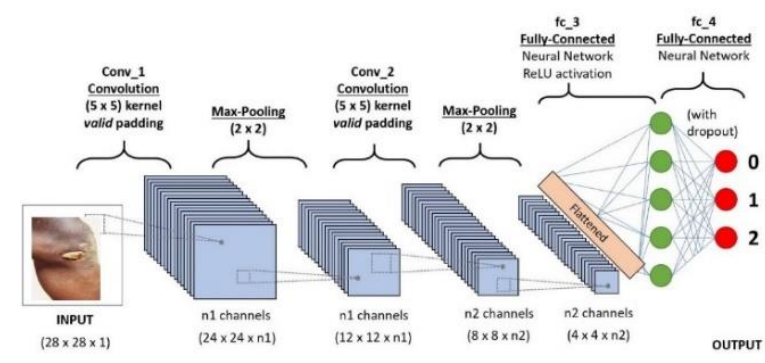

Fig.3. Proposed CNN operation

\section{B. Convolutional Neural Network (CNN)}

The Convolutional Neural Networks are deep artificial neural networks used primarily for classifying images. CNN cluster images by similarity and perform object recognition within scenes. $\mathrm{CNN}$ is applied in identifying faces, individuals, street signs, tumours, platypuses, and many other aspects of visual data [25,26]. CNN algorithm proposed in [27] was adopted for the current study. Fig. 3 shows the operational steps of $\mathrm{CNN}$. The $\mathrm{CNN}$ reduces the input image into a form which easier to process. The first convolutional layer (CL) then moves to the maxpooling layer (PL) stage second convolutional stage until 
the fully connected neural network is obtained. The combination of the CL and PL forms the $\mathrm{i}^{\text {th }} \mathrm{CNN}$.

\section{Evaluation Metrics}

The performance of the proposed model was measured using the accuracy metric as defined by equation (1) given by [17].

$$
\text { Accuracy }=\frac{T P+T F}{T P+F P+F F+T F} \times 100
$$

Where

$$
\begin{aligned}
& \mathrm{TP}=\text { number of correctly predicted Positive. } \\
& \mathrm{TF}=\text { number of correctly predicted false. } \\
& \mathrm{FP}=\text { number of incorrectly predicted positive } \\
& \mathrm{FF}=\text { number of incorrectly predicted false. }
\end{aligned}
$$

\section{RESULTS AND DISCUSSIONS}

This section presents the experimental setup, the obtained results, and its discussion.

\section{A. Experimental setup}

An experiment of the proposed skin-disease detection system was carried out to estimate its performance. An Intel Core i3 @ $3.0 \mathrm{GHz}$ with 8 GB RAM laptop was used. The proposed skin-disease detection system was implemented with Tensorflow library and Python. Fig. 4 shows the interface of the proposed method, where a user follows two simple steps to identify and skin disease. The user firstly clicks on the load file button to select an image of skin disease, then clicks on the upload image button to load the image into the system for onward processing. The system processes the image and classifies the image as Atopic dermatitis or Acne vulgaris or Scabies based on the pattern extracted, as shown in Fig. 4.

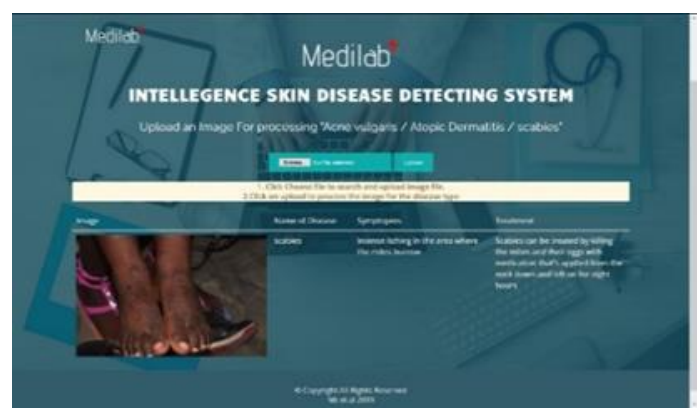

Fig.4. The interface of the proposed system for skin disease detection

\section{B. Experimental Results}

The final clean dataset was partitioned into three (3) subset, $75 \%$ for training, $15 \%$ for testing, and $10 \%$ for validation of the proposed model. Fig. 5 shows the accuracy score during the training phase. It is revealed that the proposed model obtained a training accuracy of $89 \%$.

Fig. 6 shows the graph loss rate. The loss rate declined as the efficiency increased. The results revealed an average testing accuracy measure of $88 \%$ for atopic dermatitis, $85 \%$ for acne vulgaris, and $84.7 \%$ for scabies, as shown in Fig. 7. The prediction time was 0.0010 seconds, compared with human diagnosis is many times faster.

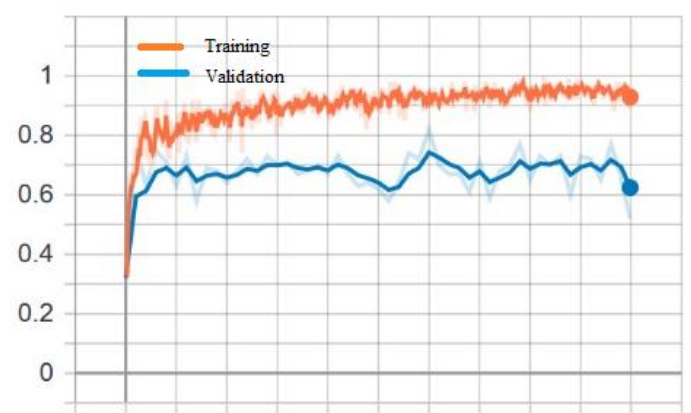

Fig.5. Accuracy of Training

The difference in accuracy level by the model over atopic dermatitis, acne vulgaris, and scabies can be attributed to the difference in data size and the difference in the quality of obtained images. The results confirm that prediction is affected by the data size and its quality.

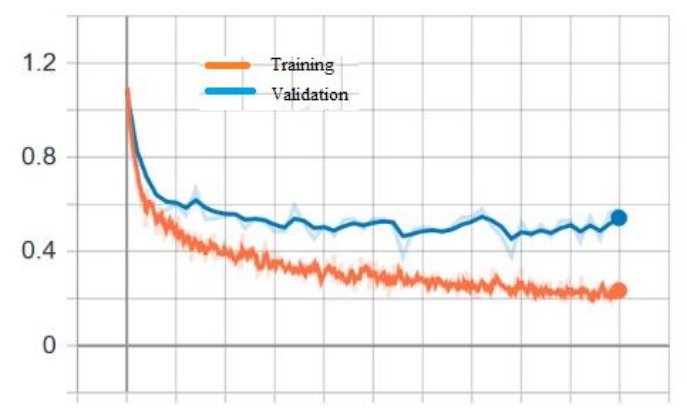

Fig. 6. Loss Rate

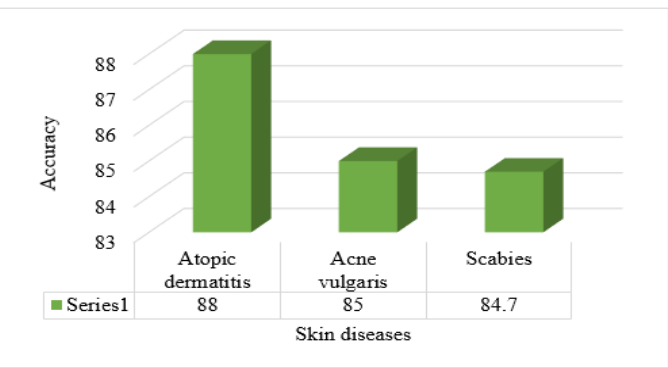

Fig.7. Accuracy Measure of the predictive model

\section{Discussion}

The current study aimed at developing a web-based skin disease detection system to help specialist and the ordinary Ghanaian detect the three (3) most common skin diseases in Ghana. The results of the current study clearly show that the proposed system offers better accuracy and faster prediction time for skin disease diagnosis as compared with the human performance rate.

The developed predictive app exhibited disease identification accuracy of $88 \%$ for atopic dermatitis, $85 \%$ for acne vulgaris, and $84.7 \%$ for scabies, with prediction 
time of less than minutes. The results revealed that technology could significantly influence the medical sector of Ghana. The accuracy measure of the current study shows that the proposed system outperforms the study of [15] (81\%) and [2] (70\%).

Few errors within $12 \%-15.3 \%$ was measured; however, as compared to human errors, the proposed system is more accurate.

Furthermore, the proposed system is capable of diagnosing these three well-known diseases with the shortest possible time of (0.0001 seconds). This achievement implies that any dermatologist, who decides to implement this study can attend to not less than 1,440 patients a day.

\section{CONCLUSION}

The current study proposed a web-based skin-disease diagnosis system, from the obtained results, it can be established that convolutional neural networks are sufficient for extracting features from raw image data for skin disease detection.

On the other hand, we do not claim our work is the first in the global health sector. However, the reduction in computational time (0.0001 seconds) of the proposed system compared with other skin diagnosis system and its ability to detect the widespread skin diseases (atopic dermatitis, acne vulgaris, and scabies) in Ghana which existing system overlooked, brings out the uniqueness and the contribution to the body of knowledge by this study. Again, the accuracy of classification $88 \%$ outperformed classification accuracy of existing studies by $(7-18) \%$.

The implementation of the proposed system on a pilot based by the Ghana health service can avoid the need for manual detection of skin disease and reduce the treatment and diagnosis time.

The researchers hoped that this study helps to catalyse the further development of artificial intelligence in dermatology service in Ghana. On the other hand, the data size for the study was limited due to the difficulty in obtaining sample data from some medical centres. Finally, authors wish to say that medilab-plus is not a final diagnostic tool; as such, it is advised that users consult their health professionals.

\section{Direction for Future research}

Future work will focus on techniques to enhance the accuracy of the classification proposed system by using hybrid machine-learning algorithms and also enable batch upload of images to multiple images to be upload at the same time for faster processing.

\section{Competing interests}

The authors of the current study declare that they have no competing interests.

\section{REFERENCES}

[1] N. Yadav, V.K. Narang, S. Utpal, Skin Diseases Detection Models using Image Processing: A Survey, Int. J. Comput. Appl. 137 (2016) 34-39.

[2] J. Rathod, V. Waghmode, A. Sodha, Diagnosis of skin diseases using Convolutional Neural Networks, in: 2nd Int. Conf. Electron. Commun. Aerosp. Technol. (ICECA 2018), IEEE Xplore, 2018: pp. 1048-1051. doi:10.1109/ICECA.2018.8474593.

[3] A. Hogewoning, A. Amoah, J.N.B. Bavinck, A. Boakye, D.; Yazdanbakhsh, M. Adegnika, S. De Smedt, Y. Fonteyne, R. Willemze, A. Lavrijsen, Skin diseases among school children in Ghana, Gabon, and Rwanda, 2019.

[4] B.E. Rosenbaum, R. Klein, P.G. Hagan, M. Seadey, N.L. Quarcoo, R. Hoffmann, M. Robinson, M. Lartey, M.C. Leger, Dermatology in Ghana: a retrospective review of skin disease at the Korle Bu Teaching Hospital Dermatology Clinic, Pan Afr. Med. J. 8688 (2017) 1-9. doi:10.11604/pamj.2017.26.125.10954.

[5] N. Codella, J. Cai, M. Abedini, R. Garnavi, A. Halpern, J.R. Smith, Deep Learning, Sparse Coding, and SVM for Melanoma Recognition in Dermoscopy Images, in: L. Zhou, L. Wang, Q. Wang, Y. Shi (Eds.), Mach. Learn. Med. Imaging, Springer International Publishing, Cham, 2015: pp. 118-126.

[6] A. Esteva, B. Kuprel, R.A. Novoa, J. Ko, S.M. Swetter, H.M. Blau, S. Thrun, Dermatologist-level classification of skin cancer with deep neural networks, Nature. 542 (2017) 115.

[7] F. Jiang, Y. Jiang, H. Zhi, Y. Dong, H. Li, S. Ma, Y. Wang, Q. Dong, H. Shen, Y. Wang, Artificial intelligence in healthcare: past, present and future, Stroke Vasc. Neurol. (2017). doi:10.1136/svn-2017-000101.

[8] A.A.L.C. Amarathunga, E.P.W.C. Ellawala, G.N. Abeysekara, C.R.J. Amalraj, Expert System For Diagnosis Of Skin Diseases, Int. J. Sci. Technol. Res. 4 (2015) 174178.

[9] M.S. Poornima, K. Shailaja, Detection of Skin Cancer Using SVM, Int. Res. J. Eng. Technol. 04 (2017).

[10] M.R.A.A.A.A. Edrees, Remote Skin Diseases Diagnosis System Using Machine Learning Techniques, University of Khartoum, 2017.

[11] Worldometers, World Population, Ghana Popul. (2019). https://www.worldometers.info/world-population/ghanapopulation/.

[12] A. Hartmann, Back to the roots - dermatology in ancient Egyptian medicine, J. Ger. Soc. Demotology. (2016) 389396. doi:10.1111/ddg.12947.

[13] G. Rosen, A history of public health, JHU Press, 2015.

[14] I. Kim, J.H. Shim, J. Yang, Face detection, 2003.

[15] SkinVision, Skin cancer: Now the most common type of cancer, (2011).

[16] Lubax, Introducing Lūbax, a smartphone-based clinical reference to identify skin lesions (2015). http://lubax.com/.

[17] I.K. Nti, A.F. Adekoya, B.A. Weyori, A systematic review of fundamental and technical analysis of stock market predictions, Artif. Intell. Rev. (2019). doi:10.1007/s10462-019-09754-z.

[18] I.K. Nti, A.F. Adekoya, B.A. Weyori, Random Forest Based Feature Selection of Macroeconomic Variables for Stock Market Prediction, Am. J. Appl. Sci. 16 (2019) 200-212. doi:10.3844/ajassp.2019.200.212. 
[19] D. Faggella, What is Machine Learning?, Tech Emerg. (2018). https://www.techemergence.com/what-ismachine-learning/ (accessed October 8, 2018).

[20] V.S. Pagolu, B. Majhi, N.R.C. Kamal, P. Ganapati, Sentiment Analysis of Twitter Data for Predicting Stock Market Movements, in: Int. Conf. Signal Process. Commun. Power Embed. Syst. (SCOPES)-2016 Sentim., 2016: pp. 1345-1350.

[21] S. Shen, J. Haomiao, Z. Tongda, Stock Market Forecasting Using Machine Learning Algorithms, CA, 2012.

[22] M. Fatima, M. Pasha, Survey of Machine Learning Algorithms for Disease Diagnostic, J. Intell. Learn. Syst. Appl. 9 (2017) 1-16. doi:10.4236/jilsa.2017.91001.

[23] I.K. Nti, G. Eric, Y.S. Jonas, Detection of Plant Leaf Disease Employing Image Processing and Gaussian Smoothing Approach, Int. J. Comput. Appl. 162 (2017) 975-8887.

[24] B. Sasan, A. Azadeh, S. Ortobelli, Fusion of multiple diverse predictors in stock market, Inf. Fusion. 36 (2017) 90-102. doi:10.1016/j.inffus.2016.11.006.

[25] D. Bogdanova, C. Santos, L. Barbosa, B. Zadrozny, Detecting Semantically Equivalent Questions in Online User Forums, Proc. 19th Conf. Comput. Lang. Learn. (2015) 123-131.

[26] S. Poria, E. Cambria, A. Gelbukh, Aspect extraction for opinion mining with a deep convolutional neural network,

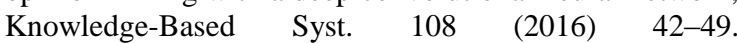
doi:10.1016/j.knosys.2016.06.009.

[27] T. Liu, S. Fang, Y. Zhao, P. Wang, J. Zhang, Implementation of Training Convolutional, ArXiv:1506.01195 [Cs.CV]. 1-10 (2015).

\section{Authors' Profiles}

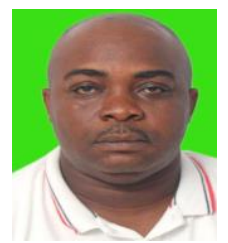

Samuel Akyeramfo-Sam holds M.Ed. Information Technology

$\mathrm{Mr}$ Akyeramfo-Sam is a Lecturer at the Department of Computer Science, Sunyani Technical University, Sunyani, Ghana. His research interests include E-Commerce, Computers in education and data security.

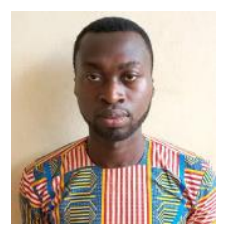

Acheampong Addo Philip is a final year student at the Department of Computer Science Sunyani Technical University, Sunyani, Ghana. Mr Acheampong is offering HND in Information Communication and Technology.

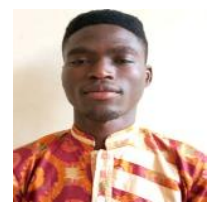

Derrick Yeboah Philip is a final year student at the Department of Computer Science Sunyani Technical University, Sunyani, Ghana. Mr Yeboah is offering HND in Information Communication and Technology

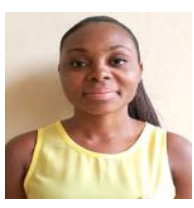

Nancy Candylove Nartey is a final year student at the Department of Computer Science Sunyani Technical University, Sunyani, Ghana. Miss. Nartey is offering HND in Information Communication and Technology

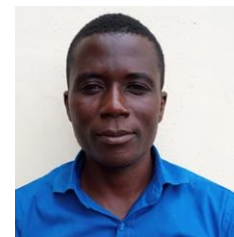

Isaac Kofi Nti holds HND in Electrical \& Electronic Engineering from Sunyani Technical University, BSc Computer Science from Catholic University College, MSc Information Technology from Kwame Nkrumah University of Science and Technology. Mr Nti is a Lecturer at the Department of Computer Science, Sunyani Technical University, Sunyani.

How to cite this paper: Samuel Akyeramfo-Sam, Acheampong Addo Philip, Derrick Yeboah, Nancy Candylove Nartey, Isaac Kofi Nti, "A Web-Based Skin Disease Diagnosis Using Convolutional Neural Networks", International Journal of Information Technology and Computer Science(IJITCS), Vol.11, No.11, pp.54-60, 2019. DOI: 10.5815/ijitcs.2019.11.06 